\title{
Infinite tension limit of the pure spinor superstring
}

\section{Nathan Berkovits}

ICTP South American Institute for Fundamental Research, Instituto de Física Teórica, UNESP - Univ. Estadual Paulista, Rua Dr. Bento T. Ferraz 271, 01140-070, São Paulo, SP, Brasil

E-mail: nberkovi@ift.unesp.br

AbStRaCT: Mason and Skinner recently constructed a chiral infinite tension limit of the Ramond-Neveu-Schwarz superstring which was shown to compute the Cachazo-He-Yuan formulae for tree-level $\mathrm{d}=10$ Yang-Mills amplitudes and the NS-NS sector of tree-level $\mathrm{d}=10$ supergravity amplitudes. In this letter, their chiral infinite tension limit is generalized to the pure spinor superstring which computes a $d=10$ superspace version of the Cachazo-HeYuan formulae for tree-level $\mathrm{d}=10$ super-Yang-Mills and supergravity amplitudes.

KeYwords: Superstrings and Heterotic Strings, Scattering Amplitudes

ARXIV EPRINT: 1311.4156 


\section{Contents}

1 Introduction 1

2 Infinite tension pure spinor superstring 2

2.1 Superparticle 2

2.2 Type II superstring 3

2.3 Scattering amplitudes 5

2.4 Heterotic superstring 5

\section{Introduction}

Although the $\mathrm{d}=10 \mathrm{~N}=1$ and $\mathrm{N}=2$ superparticle describes massless $\mathrm{d}=10$ super-YangMills and supergravity states, it is complicated to use the worldline formulation of the superparticle to compute $d=10$ super-Yang-Mills and supergravity scattering amplitudes even at tree-level. One can of course compute these amplitudes by first computing the full superstring tree-level amplitudes and then taking the infinite tension limit where $\alpha^{\prime} \rightarrow 0$, but it would be nice to have a formalism which directly computed the $\mathrm{d}=10$ massless scattering amplitudes. ${ }^{1}$ These massless tree-level amplitudes can be expressed in an elegant form using the results of Cachazo-He-Yuan [4] which generalize the $\mathrm{d}=4$ twistor-inspired formulae to arbitrary spacetime dimension.

In a recent paper [5], Mason and Skinner showed how to construct a chiral infinite tension limit of the Ramond-Neveu-Schwarz (RNS) superstring which directly computes these massless tree-level amplitudes. For the Type II version of their superstring, they verified for $\mathrm{d}=10$ supergravity states in the NS-NS sector that the Cachazo-He-Yuan formulae are correctly reproduced by their tree-level superstring scattering amplitudes. For the heterotic version of their superstring, the Cachazo-He-Yuan formulae for $\mathrm{d}=10$ Yang-Mills amplitudes are correctly reproduced but not the supergravity amplitudes. They also conjectured that their construction could be generalized to the Green-Schwarz and pure spinor formalisms of the superstring.

In this paper, the pure spinor generalization of their infinite tension limit of the superstring will be constructed and will be argued to compute all $\mathrm{d}=10$ tree-level super-YangMills and supergravity amplitudes. The construction mirrors the Mason-Skinner approach and starts with the pure spinor versions of the $\mathrm{d}=10 \mathrm{~N}=1$ and $\mathrm{N}=2$ superparticle action [6]. After replacing all worldline derivatives with antiholomorphic worldsheet derivatives, one obtains a conformally invariant worldsheet action which can be used to

\footnotetext{
${ }^{1}$ An efficient recursive method for computing these $d=10$ massless amplitudes uses the pure spinor BRST cohomology techniques developed by Mafra and collaborators in [1-3]. It would be very interesting to relate their pure spinor recursive method with the amplitudes computed by the infinite tension pure spinor superstring.
} 
compute tree-level scattering amplitudes. The BRST operator and unintegrated vertex operators are the same as in the superparticle, and the integrated vertex operators are proportional to $\bar{\delta}(k \cdot P)$ as in the Mason-Skinner integrated vertex operators.

Using the relation of pure spinors and RNS fermions, it is easy to verify that the RNS and pure spinor tree-level amplitude prescriptions agree for external Yang-Mills states and for external supergravity states in the NS-NS sector. Of course, the advantage of the pure spinor formalism is that spacetime-supersymmetry is manifest so one automatically obtains the super-Yang-Mills and supergravity amplitudes in all other sectors. Furthermore, the super-Yang-Mills and supergravity amplitudes are expessed as $d=10$ superspace versions of the Cachazo-He-Yun formulae which generalize the $\mathrm{d}=4$ supertwistor formulae and might be useful for understanding the relation of pure spinors with $\mathrm{d}=10$ supertwistors [7].

In principle, one could attempt to use this infinite tension limit of the superstring to compute $d=10$ super-Yang-Mills and supergravity loop amplitudes. However, there are two reasons to suspect this will be difficult. Firstly, d = 10 super-Yang-Mills and supergravity are not consistent quantum theories because of ultraviolet divergences. Secondly, despite the fact that the string theory describes supergravity states, the dependence on only holomorphic worldsheet variables makes it resembles an open string theory similar to the open twistor string theory of [8]. Although there exists a closed string reformulation of the open twistor string in [9], it is unclear how to compute loop amplitudes in this type of string theory, for example, if one should use annulus worldsheets [10] or torus worldsheets.

\section{Infinite tension pure spinor superstring}

\subsection{Superparticle}

The $\mathrm{d}=10 \mathrm{~N}=2$ superparticle in the pure spinor formalism is described by the worldline action $[6]$

$$
S=\int d \tau\left(P_{m} \partial_{\tau} x^{m}+p_{\alpha} \partial_{\tau} \theta^{a}+\widehat{p}_{\widehat{\alpha}} \partial_{\tau} \widehat{\theta}^{\widehat{\alpha}}+w_{\alpha} \partial_{\tau} \lambda^{\alpha}+\widehat{w}_{\widehat{\alpha}} \partial_{\tau} \widehat{\lambda}^{\widehat{\alpha}}\right)
$$

where $m=0$ to 9 are vector indices, $\alpha=1$ to 16 and $\widehat{\alpha}=1$ to 16 denote spinors of opposite chirality for the $\mathrm{N}=2 \mathrm{~A}$ superparticle and spinors of the same chirality for the $\mathrm{N}=2 \mathrm{~B}$ superparticle, $\left(x^{m}, \theta^{\alpha}, \widehat{\theta}^{\widehat{\alpha}}\right)$ are the usual variables of $\mathrm{N}=2 \mathrm{~d}=10$ superspace, $\left(P_{m}, p_{\alpha}, \widehat{p}_{\widehat{\alpha}}\right)$ are their conjugate momenta, $\lambda^{\alpha}$ and $\widehat{\lambda}^{\widehat{\alpha}}$ are bosonic pure spinor variables satisfying

$$
\lambda \gamma^{m} \lambda=0, \quad \widehat{\lambda} \gamma^{m} \widehat{\lambda}=0,
$$

and $w_{\alpha}$ and $\widehat{w}_{\widehat{\alpha}}$ are their conjugate momenta which are defined up to the gauge transformation

$$
\delta w_{\alpha}=\Lambda_{m}\left(\gamma^{m} \lambda\right)_{\alpha}, \quad \delta \widehat{w}_{\widehat{\alpha}}=\widehat{\Lambda}_{m}\left(\gamma^{m} \widehat{\lambda}\right)_{\widehat{\alpha}}
$$

Physical states are defined as ghost-number $(1,1)$ states in the cohomology of the BRST operator

$$
Q=\lambda^{\alpha} d_{\alpha}+\widehat{\lambda}^{\widehat{\alpha}} \widehat{d}_{\widehat{\alpha}}
$$


where $\lambda^{\alpha}$ and $\widehat{\lambda}^{\widehat{\alpha}}$ carry ghost-number $(1,0)$ and $(0,1)$, and $d_{\alpha}$ and $\widehat{d}_{\widehat{\alpha}}$ are the fermionic Green-Schwarz constraints defined by

$$
d_{\alpha}=p_{\alpha}+\frac{1}{2} P_{m}\left(\gamma^{m} \theta\right)_{\alpha}, \quad \widehat{d}_{\widehat{\alpha}}=\widehat{p}_{\widehat{\alpha}}+\frac{1}{2} P^{m}\left(\gamma^{m} \widehat{\theta}\right)_{\widehat{\alpha}}
$$

which satisfy the anticommutation relations

$$
\left\{d_{\alpha}, d_{\beta}\right\}=\gamma_{\alpha \beta}^{m} P_{m}, \quad\left\{\widehat{d}_{\widehat{\alpha}}, \widehat{d}_{\widehat{\beta}}\right\}=\gamma_{\widehat{\alpha} \widehat{\beta}}^{m} P_{m}
$$

It is easy to verify that $Q^{2}=0$ using the constraints of $(2.2)$ and the anticommutation relations of $(2.6)$.

The vertex operator for the $\mathrm{N}=2$ superparticle is

$$
V=\lambda^{\alpha} \widehat{\lambda}^{\widehat{\alpha}} A_{\alpha \widehat{\alpha}}(x, \theta, \widehat{\theta})=e^{i k^{m} x_{m}} \lambda^{\alpha} A_{\alpha}(\theta) \widehat{\lambda}^{\widehat{\alpha}} \widehat{A}_{\widehat{\alpha}}(\widehat{\theta})
$$

where the $\mathrm{d}=10 \mathrm{~N}=2$ superfield $A_{\alpha \widehat{\alpha}}(x, \theta, \widehat{\theta})$ has been written in momentum space and decomposed into the product of two $\mathrm{N}=1$ superfields $A_{\alpha}(\theta)$ and $\widehat{A}_{\widehat{\alpha}}(\widehat{\theta})$. The equation of motion $Q V=0$ implies that $k^{m} k_{m}=0$ and that

$$
\left(\gamma_{m n p q r}\right)^{\alpha \beta} D_{\alpha} A_{\beta}=0, \quad\left(\gamma_{m n p q r}\right)^{\widehat{\alpha} \widehat{\beta}} \widehat{D}_{\widehat{\alpha}} \widehat{A}_{\widehat{\beta}}=0
$$

where $D_{\alpha}=\frac{\partial}{\partial \theta^{\alpha}}+\frac{1}{2} k^{m}\left(\gamma_{m} \theta\right)_{\alpha}$ and $\widehat{D}_{\widehat{\alpha}}=\frac{\partial}{\partial \widehat{\theta}^{\widehat{\alpha}}}+\frac{1}{2} k^{m}\left(\gamma_{m} \widehat{\theta}\right)_{\widehat{\alpha}}$ are the $\mathrm{N}=2 \mathrm{~d}=10$ supersymmetric derivatives. And the gauge invariance $\delta V=Q \Lambda$ implies that $A_{\alpha}$ and $\widehat{A}_{\widehat{\alpha}}$ are defined up to the gauge transformations $\delta A_{\alpha}=D_{\alpha} \Lambda(\theta)$ and $\delta \widehat{A}_{\widehat{\alpha}}=\widehat{D}_{\widehat{\alpha}} \widehat{\Lambda}(\widehat{\theta})$.

In components, $A_{\alpha}(\theta)$ and $\widehat{A}_{\widehat{\alpha}}(\widehat{\theta})$ can be gauge-fixed onshell to the form

$$
\begin{aligned}
& A_{\alpha}=\frac{1}{2} a_{m}\left(\gamma^{m} \theta\right)_{\alpha}+\frac{1}{3} \xi^{\beta}\left(\gamma^{m} \theta\right)_{\alpha}\left(\gamma_{m} \theta\right)_{\beta}+\ldots \\
& \widehat{A}_{\widehat{\alpha}}=\frac{1}{2} \widehat{a}_{m}\left(\gamma^{m} \widehat{\theta}\right)_{\widehat{\alpha}}+\frac{1}{3} \widehat{\xi}^{\widehat{\beta}}\left(\gamma^{m} \widehat{\theta}\right)_{\widehat{\alpha}}\left(\gamma_{m} \widehat{\theta}\right)_{\widehat{\beta}}+\ldots
\end{aligned}
$$

where $a_{m}$ and $\widehat{a}_{m}$ are vector polarizations satisfying $k^{m} a_{m}=k^{m} \widehat{a}_{m}=0, \xi^{\beta}$ and $\widehat{\xi}^{\widehat{\beta}}$ are spinor polarizations satisfying $k_{m} \gamma_{\alpha \beta}^{m} \xi^{\beta}=k_{m} \gamma_{\widehat{\alpha} \widehat{\beta}}^{m} \widehat{\xi}^{\widehat{\beta}}=0$, and ... denotes higher-order terms in $\theta^{\alpha}$ and $\widehat{\theta}^{\widehat{\alpha}}$ which are related to the lower-order terms by BRST invariance. The polarizations of the Type II supergravity fields are expressed in terms of these unhatted and hatted super-Yang-Mills polarizations in the usual way. The polarization of the NSNS states $g_{m n}+b_{m n}+\eta_{m n} \phi$ is $a_{m} \widehat{a}_{n}$, the polarization of the R-NS gravitino and dilatino $\chi_{m}^{\alpha}+\gamma_{m}^{\alpha \beta} \rho_{\beta}$ is $\xi^{\alpha} \widehat{a}_{m}$, the polarization of the NS-R gravitino and dilatino $\widehat{\chi}_{m}^{\widehat{\alpha}}+\gamma_{m}^{\widehat{\alpha} \widehat{\beta}} \widehat{\rho}_{\widehat{\beta}}$ is $a_{m} \widehat{\xi}^{\widehat{\alpha}}$, and the polarization of the R-R bispinor field-strength $F^{\alpha \widehat{\beta}}$ is $\xi^{\alpha} \widehat{\xi}^{\widehat{\beta}}$.

\section{$2.2 \quad$ Type II superstring}

The generalization to the infinite tension limit of the superstring is obtained by simply replacing worldline $\partial_{\tau}$ derivatives in the superparticle action of (2.1) with antiholomorphic $\bar{\partial}$ worldsheet derivatives. So the infinite tension superstring action is

$$
S=\int d z d \bar{z}\left(P_{m} \bar{\partial} x^{m}+p_{\alpha} \bar{\partial} \theta^{a}+\widehat{p}_{\widehat{\alpha}} \bar{\partial} \widehat{\theta}^{\widehat{\alpha}}+w_{\alpha} \bar{\partial} \lambda^{\alpha}+\widehat{w}_{\widehat{\alpha}} \bar{\partial} \widehat{\lambda}^{\widehat{\alpha}}\right)
$$


where all conjugate momenta variables carry conformal weight $(1,0)$. Furthermore, the BRST operator is the same as in (2.4), namely

$$
Q=\int d z\left(\lambda^{\alpha} d_{\alpha}+\widehat{\lambda}^{\widehat{\alpha}} \widehat{d}_{\widehat{\alpha}}\right)
$$

where $d_{\alpha}$ and $\widehat{d}_{\widehat{\alpha}}$ are defined in (2.5). The left-moving stress tensor has vanishing central charge since $\left(x^{m}, P_{m}\right)$ contribute $+20,\left(p_{\alpha}, \theta^{\alpha}\right)$ and $\left(\widehat{p}_{\widehat{\alpha}}, \widehat{\theta}^{\widehat{\alpha}}\right)$ contribute -64 , and $\left(\lambda^{\alpha}, w_{\alpha}\right)$ and $\left(\widehat{\lambda}^{\widehat{\alpha}}, \widehat{w}_{\widehat{\alpha}}\right)$ contribute +44 .

To compute $\mathrm{N}$-point tree-level scattering amplitudes using the pure spinor formalism, one needs both unintegrated vertex operators $V$ of conformal weight $(0,0)$ and ghostnumber $(1,1)$ and integrated vertex operators $U$ of conformal weight $(1,1)$ and ghostnumber $(0,0)$. The tree-level amplitude prescription is

$$
A=\left\langle V\left(z_{1}\right) V\left(z_{2}\right) V\left(z_{3}\right) \int d^{2} z_{4} U\left(z_{4}\right) \ldots \int d^{2} z_{N} U\left(z_{N}\right)\right\rangle
$$

where the zero-mode measure factor is defined by

$$
\left\langle\left(\lambda \gamma^{m} \theta\right)\left(\lambda \gamma^{n} \theta\right)\left(\lambda \gamma^{p} \theta\right)\left(\theta \gamma_{m n p} \theta\right)\left(\widehat{\lambda} \gamma^{q} \widehat{\theta}\right)\left(\widehat{\lambda} \gamma^{r} \widehat{\theta}\right)\left(\widehat{\lambda} \gamma^{s} \widehat{\theta}\right)\left(\widehat{\theta} \gamma_{q r s} \widehat{\theta}\right)\right\rangle=1 .
$$

The unintegrated vertex operator $V(z)$ will be defined to be the same as the superparticle vertex operator of (2.7). And the integrated vertex operator $U(z)$ will be defined to be

$$
\begin{aligned}
& U(z)=e^{i k_{m} x^{m}} \bar{\delta}\left(k^{n} P_{n}\right)\left[P^{m} A_{m}(\theta)+d_{\alpha} W^{\alpha}(\theta)+\frac{1}{4}\left(\lambda \gamma_{m n} w\right) F^{m n}(\theta)\right] \\
& {\left[P^{m} \widehat{A}_{m}(\widehat{\theta})+\widehat{d}_{\widehat{\alpha}} \widehat{W}^{\widehat{\alpha}}(\theta)+\frac{1}{4}\left(\widehat{\lambda} \gamma_{m n} \widehat{w}\right) \widehat{F}^{m n}(\widehat{\theta})\right] }
\end{aligned}
$$

where $\left[A_{m}, W^{\alpha}, F^{m n}\right]$ are superfields defined in terms of $A_{\alpha}(\theta)$ as

$$
A_{m}=\frac{1}{8} \gamma_{m}^{\alpha \beta} D_{\alpha} A_{\beta}, \quad W^{\alpha}=-\frac{1}{10} \gamma_{m}^{\alpha \beta}\left(k_{m} A_{\beta}-D_{\beta} A_{m}\right), \quad F_{m n}=\frac{1}{8}\left(\gamma_{m n}\right)_{\alpha}{ }^{\beta} D_{\beta} W^{\alpha},
$$

$\left[\widehat{A}_{m}, \widehat{W}^{\widehat{\alpha}}, \widehat{F}^{m n}\right]$ are superfields defined in terms of $\widehat{A}_{\widehat{\alpha}}(\widehat{\theta})$ as

$$
\widehat{A}_{m}=\frac{1}{8} \gamma_{m}^{\widehat{\alpha} \widehat{\beta}} \widehat{D}_{\widehat{\alpha}} \widehat{A}_{\widehat{\beta}}, \quad \widehat{W}^{\widehat{\alpha}}=-\frac{1}{10} \gamma_{m}^{\widehat{\alpha} \widehat{\beta}}\left(k_{m} \widehat{A}_{\widehat{\beta}}-\widehat{D}_{\widehat{\beta}} \widehat{A}_{m}\right), \quad \widehat{F}_{m n}=\frac{1}{8}\left(\gamma_{m n}\right)_{\widehat{\alpha}} \widehat{\beta} \widehat{D}_{\widehat{\beta}} \widehat{W}^{\widehat{\alpha}},
$$

and $\bar{\delta}\left(k^{n} P_{n}\right)$ is an operator of conformal weight $(-1,1)$ defined in the same manner as in the integrated vertex operators of Mason and Skinner [5].

Note that $U(z)$ of (2.15) is manifestly spacetime supersymmetric and is gaugeinvariant under

$$
\delta A_{\alpha}=D_{\alpha} \Lambda, \quad \delta A_{m}=k_{m} \Lambda, \quad \delta \widehat{A}_{\widehat{\alpha}}=\widehat{D}_{\widehat{\alpha}} \widehat{\Lambda}, \quad \delta \widehat{A}_{m}=k_{m} \widehat{\Lambda}
$$

because of the delta function $\bar{\delta}\left(k^{n} P_{n}\right)$. Furthermore, one can verify that $Q U=0$ using the definitions of (2.16) and (2.17) and the fact that $k_{m} P^{m}=0$. One might be surprised that $U$ does not involve the terms $\partial \theta^{\alpha} A_{\alpha}(\theta)$ or $\partial \widehat{\theta}^{\widehat{\alpha}} \widehat{A}_{\widehat{\alpha}}(\widehat{\theta})$ which are present in the usual integrated supergravity vertex operator of the pure spinor formalism. However, note that these terms vanish in the superparticle (since $\partial_{\tau} \theta^{\alpha}=\partial_{\tau} \widehat{\theta}^{\widehat{\alpha}}=0$ using equations of motion) and they would violate gauge invariance and BRST invariance of $U$ because, unlike in the usual case, $Q U=0$ instead of $Q U=\partial V$. 


\section{$2.3 \quad$ Scattering amplitudes}

When the external states are in the NS-NS sector, it is straightforward to show that the pure spinor tree-level amplitude prescription of (2.13) is equivalent to the tree-level amplitude prescription using the RNS formalism of [5]. The equivalence proof is similar to the proof in the standard pure spinor formalism and uses the fact that the integrated NS-NS vertex operator of $(2.15)$ is

$$
\begin{aligned}
U=e^{i k_{m} x^{m}} \bar{\delta}\left(k^{n} P_{n}\right)\left[P^{m} a_{m}+\frac{1}{2} k_{m} a_{n}\left(\theta \gamma_{m n} p+\lambda \gamma^{m n} w\right)+\ldots\right] \\
{\left[P^{m} \widehat{a}_{m}+\frac{1}{2} k_{m} \widehat{a}_{n}\left(\widehat{\theta} \gamma_{m n} \widehat{p}+\widehat{\lambda} \gamma^{m n} \widehat{w}\right)+\ldots\right] }
\end{aligned}
$$

where $a_{m}$ and $\widehat{a}_{m}$ are the unhatted and hatted polarizations of the NS-NS state $g_{m n}+$ $b_{m n}+\eta_{m n} \phi$, and $\ldots$ denotes terms which are higher-order in $\theta^{\alpha}$ or $\widehat{\theta}^{\widehat{\alpha}}$. By $\left(p_{\alpha}, \theta^{\alpha}\right)$ and $\left(\widehat{p}_{\widehat{\alpha}}, \widehat{\theta}^{\widehat{\alpha}}\right)$ charge conservation, one can easily verify that these higher-order terms in $\theta^{\alpha}$ and $\widehat{\theta}^{\alpha}$ cannot contribute to the scattering amplitude. After dropping these higher-order terms, the vertex operator of (2.19) is identical to the vertex operator in the RNS formalism of [5] except that the pure spinor Lorentz currents $\frac{1}{2}\left(\theta \gamma_{m n} p+\lambda \gamma^{m n} w\right)$ and $\frac{1}{2}\left(\widehat{\theta} \gamma_{m n} \widehat{p}+\widehat{\lambda} \gamma^{m n} \widehat{w}\right)$ are replaced with the RNS Lorentz currents $\psi_{m} \psi_{n}$ and $\widehat{\psi}_{m} \widehat{\psi}_{n}$. Since these pure spinor and RNS Lorentz currents generate $\mathrm{SO}(9,1)$ Kac-Moody algebras of the same level [11], the OPE's of the pure spinor vertex operators of (2.19) are identical to the OPE's of the RNS vertex operators of [5].

Furthermore, note that the zero mode measure factor of (2.14) correctly reproduces the three-point supergravity amplitude when all three vertex operators are unintegrated (for the same reason as in the usual pure spinor superstring). Combining this fact with the equivalent OPE's of the integrated NS-NS vertex operators implies that the pure spinor ampitude prescription of (2.13) agrees with the RNS amplitude prescription when all external states are NS-NS, which was shown by Mason and Skinner in [5] to correctly reproduce the Cachazo-He-Yuan formulae of [4].

In addition, the pure spinor amplitude prescription of (2.13) automatically provides a manifestly supersymmetric generalization of the Cachazo-He-Yuan formulae which describes in $\mathrm{d}=10$ superspace the tree-level amplitudes of $\mathrm{d}=10 \mathrm{~N}=2$ supergravity. It would be very interesting to work out the properties of this supersymmetric formula.

\subsection{Heterotic superstring}

Finally, one can easily generalize these results to $d=10$ super-Yang-Mills by constructing the pure spinor analog of the Mason-Skinner heterotic superstring of [5]. In this case, the hatted variables of (2.11) are replaced with a holomorphic current algebra contributing central charge +16 and a set of $(b, c)$ Virasoro ghosts contributing central charge -26 . So the infinite tension limit of the pure spinor heterotic superstring action is

$$
S=\int d z d \bar{z}\left(P_{m} \bar{\partial} x^{m}+p_{\alpha} \bar{\partial} \theta^{a}+w_{\alpha} \bar{\partial} \lambda^{\alpha}+\bar{b} \bar{\partial} c\right)+S_{C}
$$

where $S_{C}$ is the worldsheet action for the current algebra. 
The BRST operator for this heterotic superstring is

$$
Q=\int d z\left(\lambda^{\alpha} d_{\alpha}+c\left(P_{m} \partial x^{m}+p_{\alpha} \partial \theta^{\alpha}+w_{\alpha} \partial \lambda^{\alpha}+T_{C}\right)+b c \partial c\right)
$$

where $T_{C}$ is the $c=16$ stress-tensor of the current algebra. And the tree-level amplitude prescription is

$$
A=\left\langle V\left(z_{1}\right) V\left(z_{2}\right) V\left(z_{3}\right) \int d^{2} z_{4} U\left(z_{4}\right) \ldots \int d^{2} z_{N} U\left(z_{N}\right)\right\rangle
$$

where $V$ and $U$ are unintegrated and integrated vertex operators in the BRST cohomology, and the zero-mode measure factor is defined by

$$
\left\langle\left(\lambda \gamma^{m} \theta\right)\left(\lambda \gamma^{n} \theta\right)\left(\lambda \gamma^{p} \theta\right)\left(\theta \gamma_{m n p} \theta\right) c \partial c \partial^{2} c\right\rangle=1 .
$$

The unintegrated vertex operator $V$ describing the super-Yang-Mills multiplet is

$$
V=e^{i k_{m} x^{m}} c \lambda^{\alpha} A_{\alpha}^{I}(\theta) J^{I}
$$

where $A_{\alpha}^{I}(\theta)$ is the super-Yang-Mills spinor gauge field, $I$ denotes the adjoint representation of the gauge group, and $J^{I}$ are the holomorphic currents of conformal weight $(1,0) . Q V=$ 0 implies that $k^{m} k_{m}=0$ and $\gamma_{m n p q r}^{\alpha \beta} D_{\alpha} A_{\beta}^{I}=0$, and $\delta A_{\alpha}^{I}=D_{\alpha} \Lambda^{I}$ under the gauge transformation $\delta V=Q\left(c \Lambda^{I} J^{I}\right)$. As in (2.9), $A_{\alpha}^{I}$ can be gauge-fixed onshell to

$$
A_{\alpha}^{I}=\frac{1}{2} a_{m}^{I}\left(\gamma^{m} \theta\right)_{\alpha}+\frac{1}{3} \xi^{I \beta}\left(\gamma^{m} \theta\right)_{\alpha}\left(\gamma_{m} \theta\right)_{\beta}+\ldots
$$

where $a_{m}^{I}$ and $\xi^{I \alpha}$ are the gluon and gluino polarizations satisfying $k^{m} a_{m}^{I}=k_{m} \gamma_{\alpha \beta}^{m} \xi^{I \beta}=$ 0 , and $\ldots$ are terms higher-order in $\theta^{\alpha}$ which are related to the lower-order terms by BRST invariance. Although one also can define the unintegrated vertex operator $V=$ $e^{i k_{m} x^{m}} c \lambda^{\alpha} A_{\alpha m}(\theta) P^{m}$, this operator does not appear to correctly describe supergravity [5].

The integrated vertex operator $U$ describing the super-Yang-Mills multiplet is defined in analogy with $(2.15)$ as

$$
U(z)=e^{i k_{m} x^{m}} \bar{\delta}\left(k^{n} P_{n}\right)\left[P^{m} A_{m}^{I}(\theta)+d_{\alpha} W^{I \alpha}(\theta)+\frac{1}{4}\left(\lambda \gamma_{m n} w\right) F^{I m n}(\theta)\right] J^{I}
$$

where $\left[A_{m}^{I}, W^{I \alpha}, F^{I m n}\right]$ are defined in terms of $A_{\alpha}^{I}(\theta)$ as in (2.16). Gauge invariance and BRST invariance of (2.26) are verified as in (2.18).

Using the same arguments as in the previous subsection, one can check that the pure spinor tree-level amplitude prescription of (2.22) reproduces the RNS tree-level amplitude prescription of [5] when all external states are Yang-Mills gluons. Since these $d=10$ Yang-Mills amplitudes were shown in [5] to coincide with the Cachazo-He-Yuan formulae of [4], the prescription of (2.22) provides a supersymmetric generalization of these formulae to $\mathrm{d}=10$ super-Yang-Mills. Hopefully, this supersymmetric generalization of twistorinspired formulae will be useful for identifying the appropriate $\mathrm{d}=10$ generalization of $\mathrm{d}=4$ supertwistors. 


\section{Acknowledgments}

I would like to thank CNPq grant 300256/94-9 and FAPESP grants 09/50639-2 and 11/11973-4 for partial financial support.

Open Access. This article is distributed under the terms of the Creative Commons Attribution License (CC-BY 4.0), which permits any use, distribution and reproduction in any medium, provided the original author(s) and source are credited.

\section{References}

[1] C.R. Mafra, Towards field theory amplitudes from the cohomology of pure spinor superspace, JHEP 11 (2010) 096 [arXiv: 1007.3639] [INSPIRE].

[2] C.R. Mafra, O. Schlotterer, S. Stieberger and D. Tsimpis, A recursive method for SYM n-point tree amplitudes, Phys. Rev. D 83 (2011) 126012 [arXiv:1012.3981] [INSPIRE].

[3] C.R. Mafra, O. Schlotterer and S. Stieberger, Complete N-point superstring disk amplitude I. Pure spinor computation, Nucl. Phys. B 873 (2013) 419 [arXiv:1106.2645] [INSPIRE].

[4] F. Cachazo, S. He and E.Y. Yuan, Scattering of massless particles in arbitrary dimension,

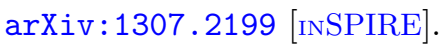

[5] L. Mason and D. Skinner, Ambitwistor strings and the scattering equations, arXiv: 1311.2564 [INSPIRE].

[6] N. Berkovits, Covariant quantization of the superparticle using pure spinors, JHEP 09 (2001) 016 [hep-th/0105050] [INSPIRE].

[7] N. Berkovits, Ten-dimensional super-twistors and Super-Yang-Mills, JHEP 04 (2010) 067 [arXiv:0910.1684] [INSPIRE].

[8] N. Berkovits, An alternative string theory in twistor space for $N=4$ super Yang-Mills, Phys. Rev. Lett. 93 (2004) 011601 [hep-th/0402045] [INSPIRE].

[9] L. Mason and D. Skinner, Heterotic twistor-string theory, Nucl. Phys. B 795 (2008) 105 [arXiv:0708.2276] [INSPIRE].

[10] L. Dolan and P. Goddard, Tree and loop amplitudes in open twistor string theory, JHEP 06 (2007) 005 [hep-th/0703054] [INSPIRE].

[11] N. Berkovits, Super Poincaré covariant quantization of the superstring, JHEP 04 (2000) 018 [hep-th/0001035] [INSPIRE]. 